\title{
Transformaciones y rasgos discursivos del spot electoral en los comicios generales del 26J en España
}

Transformations and discursive features of the campaign ads in the Spanish 26J general elections

\section{Transformações e características discursivas do anúncio eleitoral nas eleições gerais de 26] na Espanha}

Paz Villar-Hernández, Universidad de Valencia, Valencia, España (paz.villar@uv.es) NelloPellisser Rossell,Universidad de Valencia,Valencia, España (manuel.pellicer@uv.es)

RESUMEN | El spot electoral ha encontrado en las redes sociales un marco permanentemente abierto de difusión, que va más allá del espacio y del tiempo electoral regulado. En este artículo se presenta un análisis de los anuncios difundidos por los partidos con mayor representación parlamentaria (PP, PSOE, Unidos Podemos y Ciudadanos) en el Congreso de los Diputados en el período previo a las elecciones que dieron lugar a la XII Legislatura en España. Mediante el análisis descriptivo y el análisis de contenido, se examinaron los spots difundidos en YouTube para las elecciones generales del 26 de junio de 2016 por las formaciones políticas aludidas. Entre los resultados más destacados se observa un aumento en el número de anuncios políticos en los comicios de 2015 y 2016, una clara desintermediación -al dejar de lado la legislación sobre publicidad electoral vigente para otros medios- y la preponderancia de anuncios de temas programáticos sobre los de otro tipo, así como de apelaciones emotivas sobre otras.

PALABRAS CLAVE: comunicación política; spot electoral; redes sociales; producción audiovisual; publicidad electoral; desintermediación. 
ABSTRACT/Campaign ads have found in social networks a permanently open platform for dissemination that transcends the regulated space and time limits. This article presents an analysis of the ads broadcast by the parties with the highest parliamentary representation (PP, PSOE, Unidos-Podemos and Ciudadanos) in the Spanish Parliament in the period leading up to the 12th Legislature elections. Through descriptive and content analysis, we examined the ads released on YouTube for the Spanish elections of 26 June 2016 by these political groups. Among the most relevant results, we observe an increase in the number of campaign ads, clear disintermediation -by bypassing the legislation on electoral advertising which is in force of other media- and the preponderance of ads on programme issues and emotional appeals over others.

KEYWORDS: political communication; campaign ad; social networks; audiovisual production; campaign advertising; disintermediation.

RESUMO|O anúncio eleitoral encontrou nas redes sociais uma estrutura de difusão permanentemente aberta que vai além do espaço e do tempo eleitoral regulamentado. Este artigo apresenta uma análise dos anúncios divulgados pelos partidos com a mais alta representação parlamentar (PP, PSOE, Unidos Podemos e Ciudadanos) no Congresso dos Deputados no período anterior às eleições que deram origem à XII Legislatura na Espanha. Por meio da análise descritiva e da análise de conteúdo, os anúncios publicados no YouTube para as eleições espanholas do 26 de junho de 2016 foram examinados pelas formações políticas mencionadas. Entre os resultados mais destacados está o aumento do número de anúncios políticos nas eleições de 2015 e 2016, uma clara desintermediação - deixando de lado a legislação sobre publicidade eleitoral em vigor para outras mídias - e a preponderância de anúncios de temas programáticos sobre aqueles de outro tipo, bem como apelos emocionais nos outros.

PALAVRAS-CHAVE: comunicação política; anúncio eleitoral; redes sociais; produção audiovisual; propaganda eleitoral; desintermediação. 


\section{INTRODUCCIÓN}

Tras las elecciones del 20 de diciembre de 2015 (20D) se inició la XI Legislatura, la más corta hasta ese momento de la historia democrática española. Dado que ningún partido consiguió formar gobierno, en mayo se abrió un nuevo período electoral, que desembocó en las elecciones celebradas el 26 de junio de 2016.

En el marco de la sociedad en red, el video electoral se ha afianzado como una referencia troncal de las estrategias publicitarias durante las campañas, al encontrar un espacio desregulado en el que los partidos se dirigen a la ciudadanía fuera del tamiz de los medios, lo que ha provocado transformaciones en la producción y difusión del spot (Sádaba \& Jové, 2017). Desde 2008, el uso del spot electoral en redes ha sido un elemento más de las campañas, ha ganado protagonismo, ha diversificado sus modalidades discursivas y se han introducido novedosas estrategias formales y creativas (Kaid \& Holtz-Bacha, 2006; Sádaba \& Jové, 2017).

Hasta la irrupción de Internet, el espacio de difusión del spot electoral era exclusivamente el de la pantalla televisiva. Desde 1985, la Ley Orgánica 5/1985 de 19 de junio, del Régimen Electoral General (LOREG), ha regulado la publicidad electoral en España. Según esta norma, no pueden contratarse espacios de publicidad electoral en los medios de titularidad pública, ni tampoco en las emisoras de televisión privada (Ley Orgánica 2/1988, de 3 de mayo). La LOREG establece que los partidos que concurren a las elecciones tienen derecho a espacios gratuitos de propaganda electoral en los medios públicos (art. 60). Las distintas modificaciones de la LOREG, como la Ley Orgánica 13/1994, de 30 de marzo, la Ley Orgánica 8/1999, de 21 de abril, así como la Ley Orgánica 6/2002 de Partidos Políticos no introdujeron novedades sobre este aspecto, y tampoco lo hizo la reforma de una buena parte del articulado de la LOREG llevada a cabo mediante la Ley Orgánica 2/2011, de 28 de enero.

Con el desarrollo de las redes sociales, este marco regulatorio ha sido superado por la realidad, al ir la campaña más allá del tiempo y del espacio regulado, y haberse trasladado al espacio digital de las redes globales. Como señala Mattelart (2000), "las nuevas prácticas del marketing sociopolítico no viven ya sólo en los períodos calientes de los enfrentamientos entre candidatos, sino que se han convertido en un elemento que estructura la vida diaria de todo habitante de la ciudad" (pp. 111-112). Ahora las formaciones políticas están presentes en Twitter, Facebook, Instagram o YouTube, y en estos espacios gestionan el cuándo, el cómo y el público al que dirigirán sus mensajes, todo ello sin más mediación que la que supone la estructura tecnológica de las plataformas de redes (Enguix Oliver, 2017). Con la extensión del uso de estas redes, la campaña se ha abierto a todo 
tipo de participantes, simpatizantes y detractores o simplemente comentaristas y prosumers (Toffler, 1980) creando un flujo comunicativo difícilmente controlable por los equipos de comunicación de los partidos.

A este nuevo escenario ha contribuido también la accesibilidad y popularización de estos canales de difusión. Han aparecido nuevos perfiles profesionales destinados a gestionar las cuentas en las redes de los partidos y a emplear estos nuevos medios, así como la tecnología Big Data asociada para diseñar una campaña electoral, reestructurando así estrategias, prácticas y mensajes para públicos segmentados (Serazio, 2014). Estos equipos han asumido, si no toda, una parte significativa de las actividades de producción audiovisual como consecuencia de la simplificación de estos procesos y el abaratamiento de los costes de los dispositivos de captación, edición y posproducción audiovisual. Es en la campaña de 2000 cuando las formaciones políticas asumen plenamente las estrategias propias de una campaña publicitaria comercial (Sádaba \& Jové, 2017) virando todavía más hacia las fórmulas norteamericanas de hacer política.

En este contexto, el spot electoral encuentra un espacio desregulado, de "diálogo directo, sin intermediarios, entre el agente político y la opinión pública" (San Martí, 2003, p. 100). Entre estas redes, YouTube ocupa un lugar de referencia en la comunicación política audiovisual de los partidos (Carceller Cobos, 2013; Sádaba \& Jové, 2017; Vesnic-Alujevic \& Van Bauwel, 2014). Esta investigación analiza un tipo específico de video, el spot electoral, difundido para esas elecciones del 26 de junio (26J) en la red social YouTube por los cuatro partidos que obtuvieron mayor representación parlamentaria en las elecciones del 20D. Según el informe Digital News Report.es de la Universidad de Navarra, en 2020 el consumo de noticias mediante video creció considerablemente. Dos de cada tres usuarios acceden vía sitios web, en aplicaciones de medios de comunicación y redes sociales, entre las que YouTube mantiene una posición destacada. Esto ocurre también con el consumo de noticias en audio (o podcast). La desinformación es considerada un problema relevante y los encuestados responsabilizan principalmente a los políticos y partidos (Negredo et al., 2020).

\section{EL SPOT ELECTORAL: DEFINICIÓN Y ESTADO DE LA CUESTIÓN}

Los 28 anuncios difundidos bajo el título Eisenhower Answers America (1952) están considerados como el primer ejemplo de spot electoral que vio la luz en una campaña (Wood, 1990). Al igual que ocurriría más tarde con el debate electoral televisivo de Nixon contra Kennedy o la campaña electoral de Obama en 2008, marcarían un antes y un después en la comunicación política. 
El spot electoral ${ }^{1}$-ese tipo específico de video cuya finalidad última es convencer al electorado de la idoneidad de votar al candidato objeto de esa publicidad mediante diversas técnicas- ha cambiado mucho a lo largo de la historia, si bien sus características definitorias básicas se pueden concretar en las dos apuntadas por Kaid (1999): el control del mensaje y el uso de canales de comunicación de masas para su distribución. Sádaba (2003) incide en su carácter persuasivo y en el hecho de que no se trate de elementos mediatizados por los medios y los define como "mensajes políticos televisivos en campaña, eminentemente persuasivos, construidos por los propios partidos y no mediatizados por los medios de comunicación" (p. 166).

Si hasta hace unos años la televisión era el elemento central de las campañas electorales, las redes sociales se han adueñado de una parte relevante de este espacio público (Congosto et al., 2011; Costa, 2009; Gueorguieva, 2007), incidiendo en lo ya apuntado por Kaid (1999), pues el control del mensaje está más que nunca en manos de los partidos y de sus equipos de comunicación. Estos despliegan sus estrategias en redes sociales, tratando de influir y ejercer presión sobre la agenda de los medios clásicos y actuando en un espacio sobre el cual, en España, hasta el momento, no se aplica legislación electoral alguna. Son diversos los estudios que han analizado los efectos que esos mensajes tienen en redes. Algunos autores indican que han abierto canales de comunicación con el ciudadano, otros que han incrementado la participación política (Stanyer, 2005; Weber et al., 2003), y otros que inciden en el aumento de la participación juvenil (Lupia \& Philpot, 2005). Bimber (2003), sin embargo, apunta que de modo alguno influyen en el comportamiento electoral.

En España, los primeros spots electorales aparecieron en el año 1977, con motivo de las primeras elecciones democráticas ${ }^{2}$, cuando en un espacio propagandístico de hora y media, nueve partidos presentaron sus propuestas de gobierno en no más de 15 minutos cada uno. Desde ese momento, el spot ha evolucionado, sobre todo tras la introducción de Internet y las redes sociales como herramientas de comunicación política (Herrero \& Connolly-Ahern, 2004; Kaid \& Holtz-Bacha, 2006;

1. Según Herrero y Connolly-Ahern (2004) y Sádaba (2003) podemos hablar de publicidad política en España, aun tratándose del Estado, con inserciones publicitarias electorales gratuitas y sin aplicabilidad de las leyes de la publicidad a este tipo de mensaje persuasivo. Otros autores, como Canel (1999), consideran que ese término no estaría bien aplicado. Aludiremos al spot electoral, indistintamente, al referinos a publicidad electoral, publicidad política y propaganda política.

2. Ver un ejemplo en la web de RTVE, apartado "Propaganda electoral de los líderes en las elecciones de $1977 "$ (n.d.). 
Sádaba, 2003). En los últimos años se han prodigado los trabajos sobre este fenómeno publicitario-comunicativo desde la perspectiva comunicacional y empírica, que había sido relegada en los primeros estudios (Herrero \& ConnollyAhern, 2004). Así, al ya clásico trabajo de Sádaba de 2003 sobre los anuncios de los partidos políticos en televisión en España entre 1993 y 2000, se añaden otros. Peña Jiménez (2011) estudia el caso específico del spot electoral negativo, tan presente en la investigación americana o la tipología del spot electoral (Peña Jiménez \& García Jiménez, 2010). Destacan análisis longitudinales e históricos (Carceller Cobos, 2013; Connolly-Ahern \& Herrero 2006; García Beaudoux \& D’Adamo, 2007; Herrero \& Connolly-Ahern, 2004; Peña Jiménez, 2013; Sádaba \& Jové, 2017), análisis comparativos (Kaid \& Holtz-Bacha, 2006; Pineda et al., 2013), u otros más centrados en las estrategias persuasivas del spot (Capdevila, 1997; Capdevila, 2002; Gómez \& Capdevila, 2011, 2012; Capdevila et al., 2019; Peña Jiménez, 2010; Pericot \& Capdevila, 2001).

YouTube es probablemente la red social que menor investigación empírica ha generado en el ámbito político (Berrocal-Gonzalo et al., 2017; Gil-Ramírez, Castillero, \& Gómez-de-Travesedo, 2020). Sin embargo, importantes trabajos en nuestro entorno, como el de Gil-Ramírez, Gómez-de-Travesedo y Almansa-Martínez (2020), analizan el tipo de participación política en esta plataforma. Concluyen que la calidad de la argumentación política es escasa y que no hay elementos que indiquen que esta red contribuya a la calidad democrática a partir de la expresión de opiniones respetuosas y cívicas, sino más bien lo contrario. En cuanto a las estrategias de comunicación política de los partidos en YouTube a excepción de Vox, ninguno de ellos logra colocar sus producciones audiovisuales entre las más visualizadas (GilRamírez, Castillero y Gómez de Travesero, 2020), lo que afecta controlar su imagen. Por otra parte, Rodríguez-Breijo y sus colegas (2018) concluyen que la información política no es la más visualizada en esta red y, cuando se consume, se prefiere en forma de infoentretenimiento. Respecto de investigaciones sobre el 26J y YouTube, Berrocal-Gonzalo y sus colegas (2017) analizan el consumo mediático de este tipo de creación audiovisual mediante un análisis de contenido descriptivo contrastado de 80 videos distribuidos en YouTube. Subrayan la existencia de una dinámica constante en su creación en un porcentaje similar, tanto en campaña electoral como en ese otro período de "campaña permanente" (Blumenthal, 1980). Otro rasgo al que apuntan es a la personalización política, que alcanza el $87 \%$ de su muestra.

\section{MATERIAL Y MÉTODO}

Nuestro objeto de estudio es el análisis de los spots electorales difundidos en YouTube para las elecciones del 26 de junio de 2016 por los cuatro partidos con mayor 
representación parlamentaria en la XI Legislatura; es decir, el Partido Popular, el Partido Socialista Obrero Español, Podemos y Ciudadanos. Somos conscientes de que los partidos -mediante sus asociaciones locales, simpatizantes, etc.- crearon más videos de los que aquí se analizan, pero los seleccionados representan de forma específica la voz de los conglomerados.

Esta investigación intenta responder a las siguientes cuestiones:

1. Si las redes sociales han provocado un aumento en la producción de spots electorales, debido a su facilidad de difusión.

2. Si se ha producido una desintermediación (Castells, 2008; Gallardo-Paúls \& Enguix Oliver, 2016) con relación al empleo del anuncio electoral.

3. Si en las elecciones del 26J continua el cambio de tendencia apuntado por Kaid y Holtz-Bacha (2006) de reducción de la duración del spot político.

4. Siguiendo la propuesta de codificación empleada por Kaid y Holtz-Bacha (1995; 2006), nos preguntamos por la tipología de anuncio predominante en dichas elecciones del 2016, es decir:

a. Si predominan los spots que priorizan algún tema del programa electoral, respecto de la imagen de los candidatos o una combinación de ambos.

b. Si la valoración más recurrente en el corpus tiene un sentido positivo (1), negativo (o de ataque) (2), de contraste (3), o una combinación de esas tres opciones (4).

c. Si el tipo de interpelación mayoritariamente empleada es ética, lógica o emocional, o una combinación de alguna/s de ella/s.

Para responder a las tres primeras preguntas de investigación se ha recurrido a un análisis cuantitativo y descriptivo.

Dada la imposibilidad de recopilar todos los spots creados por los partidos desde las elecciones de 1996, para responder a la primera pregunta se realizó un análisis documental y se localizaron las fuentes bibliográficas que habían analizado el spot electoral en España en las bases de datos de Web ofScience, Scopus, Dialnet y Google Scholar, estableciendo como criterios de búsqueda "spot electoral" y "España”, así como también los mismos términos en lengua inglesa. Dada la escasez de resultados, ese análisis se completó con otras referencias obtenidas en búsquedas paralelas. Algunas de las investigaciones hacían referencia en sus datos al universo muestral y otras a la muestra empleada. Los datos de 2015 y 2016 fueron recopilados por los autores. 
El análisis de contenido de los spots electorales nos permite responder a la cuarta pregunta formulada. En esta, se siguieron los pasos apuntados por Igartúa Perosanz (2006) para un adecuado empleo de la técnica, si bien para la conceptualización recurrimos al modelo de codificación propuesto por Kaid y Holtz Bacha (1995, 2006), ampliamente utilizado en la investigación latinoamericana sobre el spot electoral (García Beaudoux et al., 2007) al entender que respondía adecuadamente a la complejidad del corpus analizado.

Son varias las propuestas tipológicas sobre el spot político consideradas en este estudio (Benoit, 1999; Devlin, 1989; Diamond \& Bates, 1984; Kaid \& Sanders, 1978), si bien hemos hecho uso fundamentalmente de la planteada por Benoit (1999). Este, diferencia entre una función positiva versus negativa y una temática del spot basada en la imagen del candidato versus los temas de campaña. El autor prefiere hablar de anuncios de política (o policy) porque entiende que ambos aspectos, issues e images, están muy interrelacionados y no responden de forma exclusiva a la temática del anuncio, aunque es consciente de la extensa penetración de aquellos conceptos en el ámbito de la comunicación política.

Kaid (2004) señala esa falsa dicotomía subrayada por Benoit entre asuntos e imágenes y el problema que implica la posibilidad de multicodificación de un mismo campo. Para eludir esta cuestión, empleamos las categorías teóricas propuestas por los investigadores de la Annenberg School for Communication de la Universidad de Pennsylvania, que gozan de gran consenso (Bartels, 1988, en García Beaudoux \& D’Adamo, 2007), y que distinguen entre anuncios positivos, de contraste o negativos. Como anuncios positivos analizamos aquellos que enfatizan las cualidades del candidato; como negativos, los centrados en las debilidades del oponente político, bien sea el partido o el candidato, y como de contraste, los que comparan entre uno y otro partido, candidato o planteamientos políticos.

La segunda codificación, relativa a los anuncios según sean de asunto (o propuesta) o de imagen, considera que los primeros enfatizan en el contenido las posiciones políticas del partido o del candidato, mientras los segundos muestran la imagen del candidato como epítome de sus cualidades personales, políticas, así como su experiencia y valía para ocupar la presidencia del gobierno.

En el caso de las apelaciones empleamos la clasificación de apelación lógica cuando el anuncio recurre claramente a evidencias para fundamentar sus razonamientos mediante estadísticas, datos, etc. y la apelación ética cuando el anuncio trata de enfatizar la credibilidad de la fuente para reforzar su mensaje. Por último, la apelación emocional es cuando el anuncio recurre a sentimientos o emociones para generar el efecto político deseado. 


\begin{tabular}{ccccc} 
Partido & $\begin{array}{c}\text { Título } \\
\text { identificativo }\end{array}$ & $\begin{array}{c}\text { Énfasis } \\
\text { narrativo }\end{array}$ & $\begin{array}{c}\text { Valoración } \\
\text { del spot }\end{array}$ & Tipo de apelación \\
\hline PP & Tema & Positivo & Ética \\
\hline PSOE & Imagen & Negativo & Lógica \\
\hline C's & Ambos & Comparativo & Emoción \\
\hline $\begin{array}{c}\text { Unidos } \\
\text { Podemos }\end{array}$ & & & $\begin{array}{c}\text { Combinación de alguna } \\
\text { de las anteriores }\end{array}$ \\
\hline
\end{tabular}

Tabla 1. Ficha empleada para el análisis de datos

Fuente: Elaboración propia.

Para seleccionar la muestra se localizaron las cuentas oficiales en YouTube de los cuatro partidos (Partido Popular, PSOE, Podemos, Ciudadanos) y se identificaron los videos distribuidos desde que se anunciaron elecciones. La campaña comenzó el 10 de junio y se extendió hasta la medianoche del día 24, aunque la precampaña se inició antes. La muestra se tomó en marzo de 2017, la codificación de los datos se realizó durante 2018 y los resultados se procesaron en noviembre de 2019.

El corpus definitivo lo forman 66 videos 3 : 44 spots del Partido Popular (PP), 12 del Partido Socialista Obrero Español (PSOE), 5 de Ciudadanos (C's) y 5 de Unidos Podemos (UP) ${ }^{4}$. Los datos se recogieron en una matriz de Microsoft Excel y se procesaron en SPSS.

Para recoger la información, dos codificadores independientes utilizaron una plantilla de análisis (tabla 1).

Para la cuarta pregunta de investigación se realizó una prueba de fiabilidad intercodificador Kappa de Cohen para todas las variables analizadas. En el caso de la variable énfasis narrativo, el índice de acuerdo entre codificadores fue de ,93; para la variable valoración, de ,82; y ,79 para la variable tipo de apelación. Su proximidad a 1 apunta un elevado acuerdo entre ambos codificadores en todos los casos.

\section{RESULTADOS}

Para responder a la primera pregunta de investigación, se realizó una búsqueda bibliográfica de autores que estudiaron el spot electoral entre 1996 y 2016 y el universo muestral o muestra que analizaron (tabla 2).

3. Se incluyen videos creados para la campaña electoral anterior, pues se redifundieron.

4. Unidos Podemos participó como coalición (Podemos, Izquierda Unida, Equo y CLIAS). 


\begin{tabular}{|c|c|c|c|c|c|c|c|c|}
\hline Bibliografía & & 1996 & 2000 & 2004 & 2008 & 2011 & 2015 & 2016 \\
\hline \multirow{3}{*}{ Sádaba (2003) } & PP & 4 & 4 & & & & & \\
\hline & PSOE & 4 & * & & & & & \\
\hline & IU & 4 & * & & & & & \\
\hline \multirow{2}{*}{$\begin{array}{l}\text { Herrero y Connolly- } \\
\text { Ahern (2004) }\end{array}$} & PP & & 24 & & & & & \\
\hline & PSOE & & * & & & & & \\
\hline $\begin{array}{l}\text { Connolly-Ahern \& y } \\
\text { Herrero (2007) }\end{array}$ & PP & 12 & & & & & & \\
\hline \multirow{3}{*}{$\begin{array}{l}\text { García-Beaudoux \& } \\
\text { D'Adamo (2007) }\end{array}$} & PP & & & 1 & & & & \\
\hline & PSOE & & & 2 & & & & \\
\hline & IU & & & 2 & & & & \\
\hline \multirow{2}{*}{ Carceller Cobos (2013) } & PP & & & 4 & 9 & 10 & & \\
\hline & PSOE & & & 1 & 7 & 10 & & \\
\hline \multirow{3}{*}{$\begin{array}{c}\text { Gómez \& Capdevila } \\
\text { (2011) }\end{array}$} & PP & & & & 4 & & & \\
\hline & PSOE & & & & 9 & & & \\
\hline & IU & & & & 7 & & & \\
\hline \multirow{3}{*}{ Capdevila (2002) } & PP & & 15 & & & & & \\
\hline & PSOE & & 10 & & & & & \\
\hline & IU & & 5 & & & & & \\
\hline \multirow{3}{*}{$\begin{array}{c}\text { Gómez y \& Capdevila } \\
\text { (2012) }\end{array}$} & PP & & & & 15 & 4 & & \\
\hline & PSOE & & & & 12 & 4 & & \\
\hline & IU & & & & 11 & 2 & & \\
\hline \multirow{5}{*}{$\begin{array}{l}\text { Pellisser, Villar y Enguix } \\
\text { (2017) }\end{array}$} & PP & & & & & & 58 & 44 \\
\hline & PSOE & & & & & & 27 & 12 \\
\hline & IU & & & & & & 27 & \\
\hline & Podemos & & & & & & 5 & 5 \\
\hline & $C^{\prime} s$ & & & & & & 11 & 5 \\
\hline
\end{tabular}

[*] Nota: los autores no precisan cifra.

Tabla 2. Recopilación documental de los spots de las elecciones generales en España (1996-2016)

Fuente: Elaboración propia.

Ante las discrepancias cuantitativas existentes entre los artículos de idénticas elecciones, utilizamos como referencia de cada anualidad la cifra más elevada, al entender que se aproxima más al universo de análisis (tabla 3). 


\begin{tabular}{c|c|c|c|c|c|c|c} 
Partidos & $\mathbf{1 9 9 6}$ & $\mathbf{2 0 0 0}$ & $\mathbf{2 0 0 4}$ & $\mathbf{2 0 0 8}$ & $\mathbf{2 0 1 1}$ & $\mathbf{2 0 1 5}$ & $\mathbf{2 0 1 6}$ \\
\hline PP & 12 & 24 & 4 & 15 & 10 & 58 & 44 \\
\hline PSOE & 4 & 10 & 2 & 12 & 10 & 27 & 12 \\
\hline Podemos & & & & & & 27 & 5 \\
\hline C's & & & & & & 5 & 5 \\
\hline IU & 4 & 5 & 1 & 11 & 2 & 11 & \\
\hline Total & 20 & 39 & 7 & 38 & 22 & 128 & 66 \\
\hline
\end{tabular}

[1] Nota: C's y Podemos fueron creados en 2006 y 2014, respectivamente. En las elecciones de 2016, IU se presentó con Podemos en la coalición Unidos Podemos.

Tabla 3. Spots creados por los partidos políticos durante varias elecciones generales en España

Fuente: Elaboración propia.

Respecto de la primera pregunta, se observa que el número de spots se ha incrementado considerablemente en las dos últimas citas electorales (2015 y 2016) (tabla 3). Hasta 2011, el número global se mantenía por debajo de los 40 , cifra que en 2015 alcanza los 128, casi el doble de los producidos para el 26J. El descenso de la cifra de spots de 2016 con respecto a 2015 puede explicarse por la proximidad de ambas citas electorales, lo que provocó la reutilización de videos de 2015.

En las elecciones del 26J, el PP es el partido que más videos publicitarios difundió en YouTube $(66,7 \% ; n=44)$, seguido a gran distancia del PSOE $(18,2 \%, n=12)$ y de UP y C's (ambos con un 7,6, n= 5 respectivamente). El PP no solamente realiza y difunde más mensajes, sino que es el que más minutos dedica a esas producciones (sus 44 spots suman un total de 55'14", casi el triple del tiempo del PSOE (14'), UP (10'49") y C's (10'10").

Para valorar la desintermediación comunicativa, analizamos la difusión de los spots en diferentes momentos electorales: antes de la convocatoria de elecciones, la precampaña (3 de mayo - 9 de junio) y la campaña (10-24 de junio). Las fechas consideradas para el análisis son las del día en que se subieron a YouTube. Los datos que arroja este análisis (figura 1) muestran que la difusión se lleva a cabo principalmente fuera del período de la campaña electoral. Los spots comienzan a emitirse desde que se vislumbra la posibilidad de repetición electoral. En la investigación de BerrocalGonzalo y sus colegas (2017) este porcentaje era similar en ambos períodos.

El PP es también el partido que más spots electorales emite en los tres momentos políticos analizados y el único que lo hace antes de la convocatoria electoral. En general, y teniendo en cuenta todos los partidos analizados, la mayor parte se difunde durante la precampaña (hasta un 53\%), en campaña $(42,4 \%) \mathrm{y}$, en mucha menor medida, antes de la convocatoria de elecciones. 


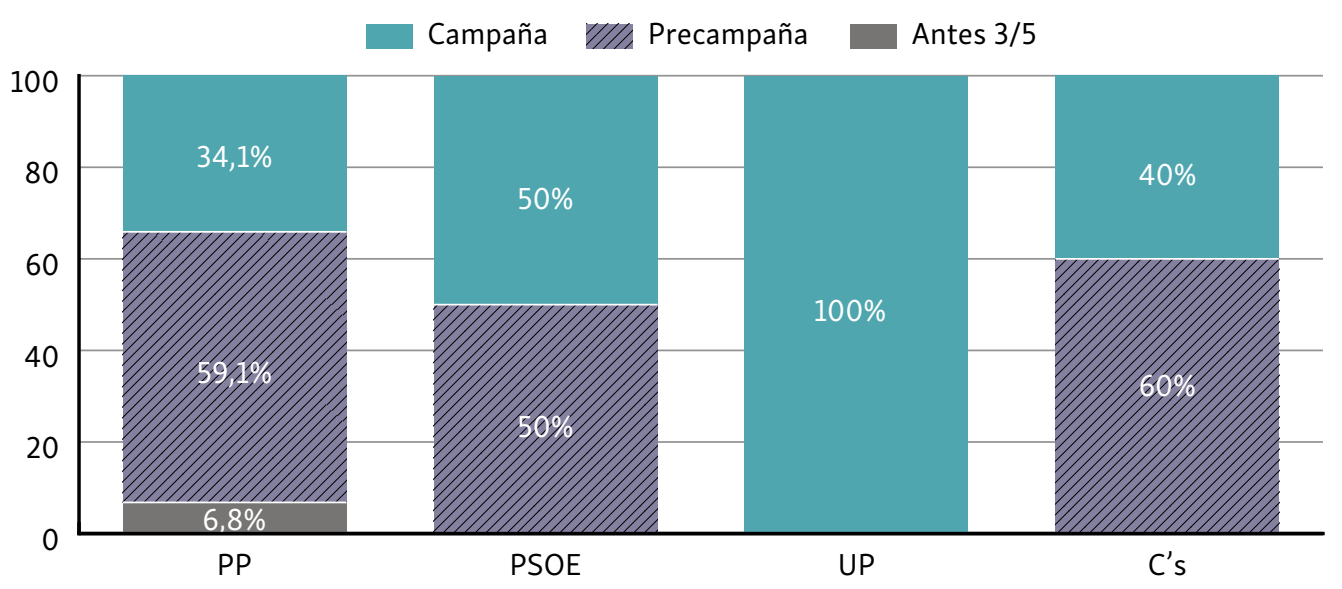

Figura 1. Período de difusión de los spots analizados

Fuente: Elaboración propia.

Se observa así que el período preelectoral es el momento de mayor actividad publicitaria (figura 1), tendencia seguida porla mayoría de los partidos, a excepción de UP. Tan solo este partido esperó al período de campaña para emitir sus spots, si bien en ello pudo influir el hecho de que concurrió a esta cita electoral en coalición con otros partidos, alianza que no se confirmó hasta el 13 de mayo.

En relación con la duración de este tipo de video electoral, Kaid y Holtz Bacha (2006) apuntan a cambios en los últimos años. Los anuncios pasaron de una media de 3-4 minutos al modelo norteamericano de 30 segundos. Nuestra investigación lo confirma con algunos matices, pues los partidos clásicos, PP y PSOE, emplean duraciones más breves (tabla 4). Ambos conglomerados optan principalmente por spots de entre 31 segundos y 1 minuto (PP n= 15 y PSOE $n=6$ ). En este sentido, son las nuevas organizaciones políticas, es decir UP y C's, las que claramente optan por videos de más de 1' (tabla 4).

Por último, analizamos cuestiones relativas al estilo audiovisual (videostyle). De entre los tres aspectos observados, los resultados relativos a la preponderancia de anuncios de propuesta o temas versus de imagen, o su combinación, se pueden ver en la figura 2. Siguiendo a García Beaudoux y D’Adamo (2007) y Kaid y HoltzBacha (2006), aunque discrepando con lo observado por Pineda y sus colegas (2013), durante el 26J los partidos plantearon mayoritariamente anuncios de propuesta/ tema $(n=48)$ frente al cultivo de la imagen del candidato $(n=12)$, o una combinación de ambas opciones $(n=6)$. Tan solo C's combina ambas opciones $(n=4)$ antes que plantear exclusivamente anuncios de temas $(n=1) y$, curiosamente, ninguno de los partidos jóvenes apuesta por la imagen de sus candidatos como único reclamo en el spot (figura 2). 
Partidos

Duración del anuncio

\begin{tabular}{ccccc} 
& $\mathbf{0 - 3 0} \mathbf{~ s .}$ & $\mathbf{3 1} \mathbf{~ s - 1 ~ \mathbf { ~ } .}$ & $\mathbf{1 : 0 1} \mathbf{- 2} \mathbf{~ m}$ & Más de $\mathbf{2} \mathbf{~}$. \\
\hline PP & 22,7 & 34,1 & 27,3 & $\mathbf{1 5 , 9}$ \\
\hline PSOE & 16,7 & 50,0 & 25,0 & 8,3 \\
\hline UP & & & 60,0 & 40,0 \\
\hline C's & & & 40,0 & 60,0 \\
\hline Total & 18,2 & 31,8 & 30,3 & 19,7 \\
\hline
\end{tabular}

Tabla 4. Duración del anuncio, en porcentaje

Fuente: Elaboración propia.

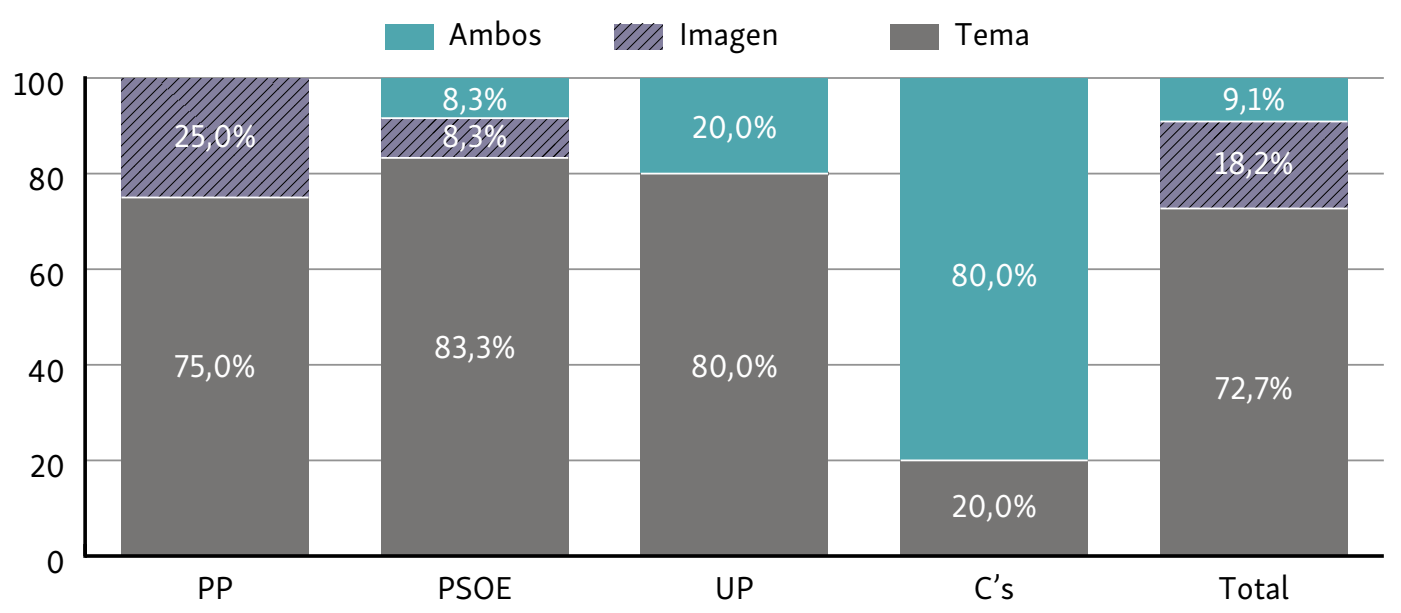

Figura 2. Spots según preferencia programática o temática

Fuente: Elaboración propia.

Respecto a la valoración del anuncio electoral, los resultados confirman la tendencia ya apuntada por Kaid y Holz-Bacha (2006) y García Beaudoux y D'Adamo (2007): la preferencia de los partidos por el anuncio positivo (C's $n=3$; PSOE $n=7 ; P P$ $n=22$ ). La excepción es UP, cuya campaña audiovisual tiene un enfoque ligeramente más negativo (solo un spot es positivo y otro comparativo (figura 3). También el PP muestra un elevado número de spots negativos o de ataque (hasta un $n=16$ ).

Nuestro análisis indica que el énfasis en las apelaciones emocionales es mayoritario en todos los partidos (UP, n= 4; PSOE, $n=7$, yP, $n=18$ ), a excepción de C's $(n=1)$. Esta última formación opta por una estrategia diferente, que prioriza la combinación de apelaciones $(n=4)$, entre las que se encuentra la emoción, pero no de forma exclusiva. Solo los partidos clásicos, PP y PSOE, emplean la apelación lógica ( $n=17$ y PSOE, $n=2$ ) o ética (PP, n= 3, y PSOE, $n=2)$, pero ni UP ni C's se decantan por un anuncio exclusivamente de ese tipo, como se puede observar en la figura 4. 


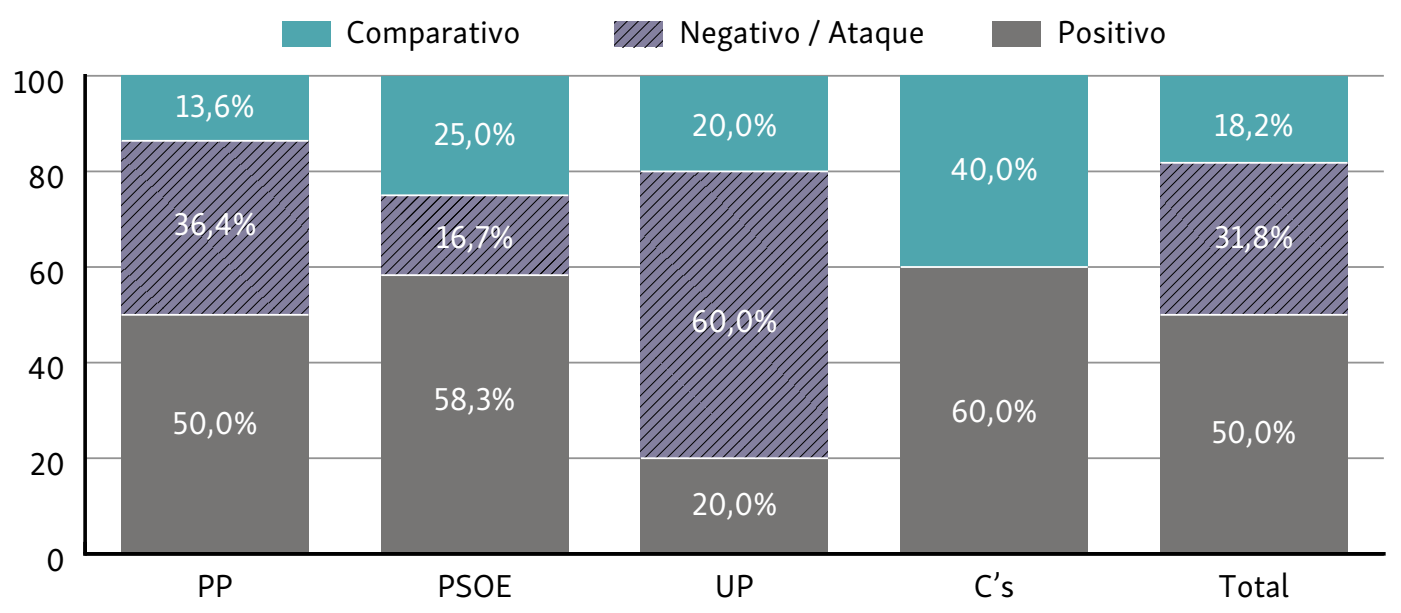

Figura 3. Spots relativos a la valoración realizada en el anuncio

Fuente: Elaboración propia.

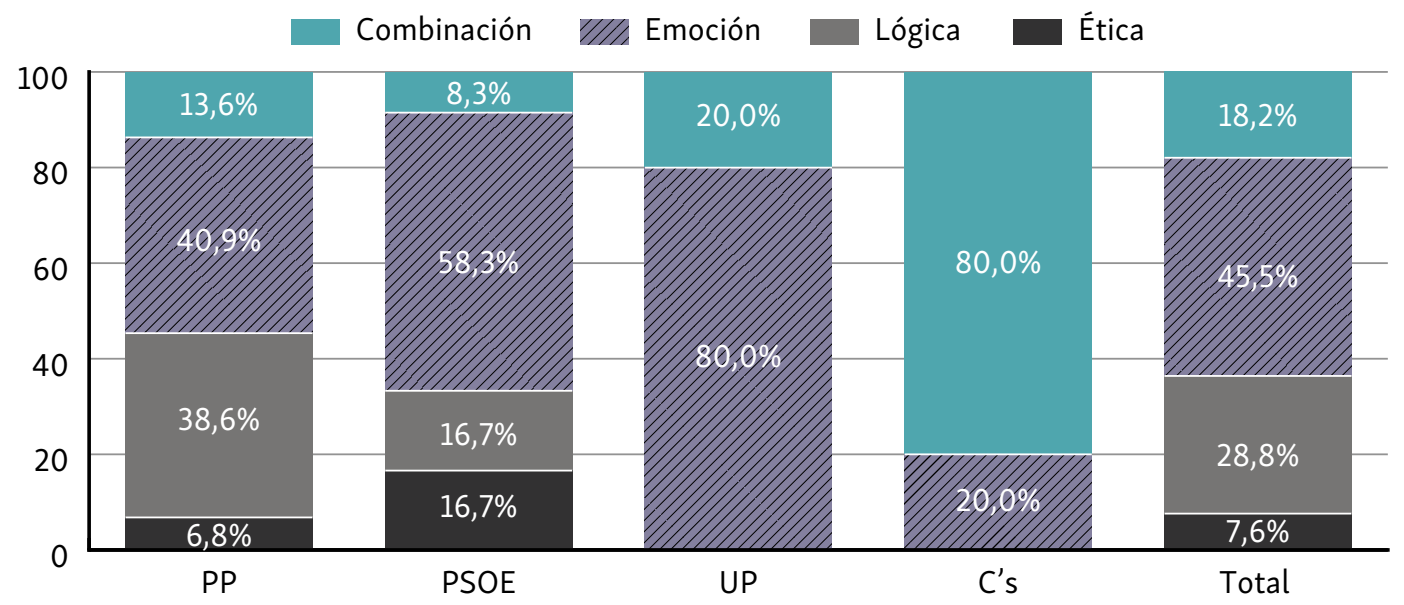

Figura 4. Tipo de apelaciones en el anuncio

Fuente: Elaboración propia.

\section{DISCUSIÓN Y CONCLUSIONES}

Señala Innerarity (2016) que "es indudable que Internet está modificando nuestras prácticas políticas" y que, aunque: “[T]odavía no sabemos con exactitud cómo será la política en la era de las redes, (...) sí estamos en condiciones de adivinar algunas de las posibilidades que inaugura y, sobre todo, qué tipo de prácticas políticas carecerán de sentido en la nueva república digital" (p. 4).

Esta investigación exploró los cambios producidos en algunas de estas prácticas, específicamente el comportamiento de los partidos políticos con mayor representación parlamentaria en España en su estrategia publicitaria electoral en YouTube. 
Con respecto a la primera de las cuestiones analizadas, observamos un aumento claro en el número de spots difundidos en las citas electorales del 26J y el 20D. Dicho incremento es evidente a partir de 2008, cuando Internet irrumpe con fuerza en la comunicación política española (Peytibi et al., 2008; Sádaba \& Jové, 2017). El número de spots creados y difundidos por los partidos el 26J señala dos tendencias: una de mayor actividad, marcada por PP y PSOE, y otra menor de las nuevas formaciones políticas, UP y C's. Sin embargo, revisados los datos de la campaña anterior, 2015, observamos que al primer grupo se uniría Podemos que, con Izquierda Unida, suma ese año 38 spots, dato por encima incluso del PSOE. Quizás la tardía conformación en 2016 de la coalición electoral con la que Unidos Podemos acudió a las elecciones ralentizó su producción de spots, a la espera de otras decisiones. Podríamos así decir que los partidos con mayor trayectoria política difunden más spots en YouTube; probablemente los recursos humanos y económicos aún son un factor determinante para crear este tipo de producciones.

Por otro lado, el empleo de redes sociales ha supuesto un incremento en el número de spots difundidos, que ya no quedan limitados a su emisión en televisión durante el período establecido por el marco legislativo vigente. Deberemos seguir observando esta tendencia, para ver si incide en modificar la legislación española respecto de este nuevo escenario digital, que parece identificarse con el de una campaña permanente (Blumenthal, 1980). Así, la precampaña electoral se convierte de hecho en un momento muy relevante, tanto como la campaña electoral (BerrocalGonzalo et al., 2017).

Aunque sabemos que no hay relación directa entre el tamaño del partido y el impacto de sus spots en la opinión pública, o entre este impacto y el porcentaje de voto obtenido (Sádaba \& Jové, 2017), también sabemos que "la exposición a la publicidad política puede afectar significativamente la calificación de la imagen de un líder" (Kaid \& Holtz-Bacha 2006, p. 454), sea esta afectación positiva o negativa. De ahí el especial interés de los partidos en crear este tipo de contenidos y difundirlos extensamente en diferentes redes sociales y entre colectivos ciudadanos. Fruto de estas transformaciones se confirma la existencia de una desintermediación favorecida por el desarrollo de las redes multimodales (Serazio, 2014), en las que son los partidos los que se comunican con el público para tratar de generar una opinión pública favorable a sus fines, independientemente de que los medios clásicos posteriormente se hagan eco de esos mensajes electorales. Siguiendo a Pericot y Capdevila (2001), los partidos y sus equipos de estrategia electoral y comunicación deciden cuándo y cómo difundir esos anuncios, qué cantidad de mensajes se producen y los contenidos sobre los que quieren incidir. 
En tercer lugar, se constata que la duración del spot electoral en España se reduce, tendencia apuntada en investigaciones anteriores, como la de los dos minutos en el año 2000 (Sádaba, 2003), o Kaid y Holtz-Bacha (2006), y que confirma la adaptación española al modelo norteamericano. En nuestra investigación se observan dos tendencias: la apuesta de los partidos clásicos por anuncios de menos de 1 minuto y la de las nuevas organizaciones, por una mayor duración. Según Sádaba (2003), “las investigaciones han demostrado que los anuncios más largos no son más efectivos, sino más bien lo contrario" (p. 177); así, partidos con mayor trayectoria histórica y gubernamental siguen la tendencia marcada por la comunicación política. Este fenómeno participa de la cultura clip (Lipovetsky, 1990) que caracteriza el relato audiovisual sea cual sea su formato. Como señala Gavaldà (1998), "el registro publicitario constituye un principio de homogeneización del flujo audiovisual, del heterogéneo palimpsesto genérico audiovisual" (pp. 182-183). El desarrollo y protagonismo de las redes sociales en el campo de la comunicación política ha extendido esta particularidad discursiva al conjunto de la publicidad electoral.

Con respecto a la cuarta de las cuestiones, concluimos que para el 26J los partidos españoles plantearon mayoritariamente anuncios de propuesta y tema, con la única excepción de C's. Seguimos así la tendencia de países como Francia, Italia y Gran Bretaña puesta de manifiesto en las elecciones españolas de 1996 y 2000 (Kaid \& Holtz-Bacha, 2006; Herrero \& Connolly-Ahern 2004) o las de 2004 (García Beaudoux \& D’Adamo, 2007) y otros países más alejados, como Polonia, Rusia o los Estados Unidos. Sin embargo, en España esto no siempre ha sido así. Por ejemplo, en las elecciones de 1996, el PSOE -en el gobierno en esos momentosrecurrió fundamentalmente a la figura de su candidato, Felipe González (Sánchez Aranda et al., 1997, en Sádaba, 2003).

Los líderes están presentes, pero no de forma arrolladora, lo que contrasta con la tendencia a la personalización política apuntada por Berrocal-Gonzalo y sus colegas (2017) en los videos políticos no producidos por las formaciones políticas. Es reseñable que ninguna de las nuevas formaciones produzca anuncios donde el candidato a presidente sea el centro de atención, a diferencia de los partidos clásicos. Ciudadanos fue el único en preferir combinar ambos elementos: imagen y tema. Curiosamente, estos usos audiovisuales contrastan con su discurso político en Twitter, donde los mensajes de las cuentas de los partidos y sus representantes inciden en los indicadores retóricos de personalización, principalmente en el caso de los partidos más jóvenes y sus líderes (Gallardo-Paúls \& Enguix-Oliver, 2016).

En cuanto al tipo de anuncio según la valoración realizada en este, los resultados confirman la preferencia de los partidos por el anuncio positivo, a excepción de UP. El escaso empleo de spots de carácter negativo había sido apuntado por Kaid y Holz-Bacha (2006) y García Beaudoux y D’Adamo (2007) para otras elecciones 
españolas. También el PP, en menor medida, incide en el mensaje negativo o de ataque, siguiendo así en el spot una tendencia ya apuntada en el análisis de sus argumentarios (Gallardo-Paúls, 2014).

Estudios sobre la comunicación política en general (García Beaudoux \& D’Adamo, 2006), y española en particular, señalan un incremento en la emotivización, lo que tiene su traslación directa en el anuncio político (Kaid \& Holtz-Bacha, 2006). Los motivos que alientan el uso de estos enfoques expresivos o emotivos en el discurso político se basan en la aceptación generalizada de que las emociones "ayudan a la gente a recordar los mensajes políticos. El miedo, la ansiedad o la ira permiten a los candidatos enfatizar valores consensuados, lo que facilita la movilización de los electores" (García Beaudoux \& D’Adamo, 2006, p. 93). Esta inclinación por el lado emotivo de la argumentación política encuentra en las redes sociales un ámbito óptimo de desarrollo, que contribuye a consolidar escenarios más pseudopolíticos que realmente políticos (Gallardo-Paúls \& Enguix-Oliver, 2016), debido en parte a rasgos propios de la estructura tecnológica, como la inmediatez, la infinitud (falta de principio y final) o la brevedad.

Como vimos en los resultados, las apelaciones emocionales en el spot fueron mayoritarias tan solo con una excepción, Ciudadanos, que, sin embargo, apostó por un conjunto de apelaciones entre las que la emoción en muchos casos estaba presente. Kaid y Holtz-Bacha (2006) identificaron esa misma tendencia en las elecciones españolas de 1996 y de 2000 (también para estas últimas, Herrero \& Connolly-Ahern, 2004). Sin embargo, una revisión histórica permite ver que esto no siempre fue así (Carceller Cobos, 2013; García Beaudoux \& D’Adamo, 2007). De hecho, esta ha sido una cuestión cambiante, en la que seguramente el contexto electoral y el diseño de campaña planteado han sido determinantes. Aunque esa emotivización del spot ha crecido en las democracias occidentales en los últimos años (Jerit, 2004; Kaid \& Holtz-Bacha, 1995, 2006) existen diferencias geográficas. Analizar cada elección y partido en su contexto permite ir a saber si podemos hablar de una tendencia generalizada a la emotivización.

En cuanto a la apelación lógica, por un lado, y ética, por otro, ambas son utilizadas mayoritariamente por los partidos clásicos (PP y PSOE), aunque en menor medida que la emoción. Probablemente, el hecho de contar con una labor de gobierno previa, que arroja datos y cifras de gestión, les permite convertir esa realidad en un instrumento de persuasión.

Como hemos visto, este artículo profundiza en un elemento de la comunicación política, el spot electoral, escasamente analizado en España, y lo hace sobre unas elecciones especialmente significativas, cuando los votantes acudieron a las urnas con un bipartidismo quebrado y una situación política de elevada crispación. 
Los resultados indican que en esos comicios y los del año anterior, 2015, los partidos incrementaron enormemente la emisión de spots electorales en YouTube; también, que las limitaciones establecidas por el marco legislativo quedaron superadas por un ecosistema comunicativo en el que las redes sociales lo trastocaron todo. A diferencia de los spots de otros comicios, en estos los partidos incidieron más en los temas políticos y mostraron en menor medida al líder como protagonista, presentaron contenidos en clave sobre todo positiva y mostraron una tendencia a la emotivización.

Esta investigación continúa la línea de trabajo sobre el spot electoral en España desarrollada por García-Beaudoux y D’Adamo (2007), Herrero y Connolly-Ahern (2004), y Kaid y Holz Bacha (2006), entre otros, y la actualiza según los datos de los comicios de 2016, para indicarnos cierta continuidad en el empleo del anuncio político basado en temas y la predominancia de la apelación emotiva.

A la vista de la consistencia y variedad del corpus recogido, se considera pertinente continuar esta investigación con un análisis del contenido discursivo del spot electoral de estas elecciones y su proceso de producción, en el que se han detectado en una primera aproximación rasgos de fragmentación discursiva, serialización e hibridación, así como un uso frecuente del reciclaje y la reutilización audiovisual.

\section{FINANCIAMIENTO}

PRODISNET-02.2019/2020: Procesos discursivos en internet: desplazamientos enunciativos y efectos hiperbólicos en el discurso político. RTI2018-093523-B-100, del Ministerio de Ciencia, Innovación y Universidades de España.

\section{REFERENCIAS}

Benoit, W. L. (1999). Seeing spots: a functional analysis of presidential television advertisements, 1952-1996. Praeger.

Berrocal Gonzalo, S., Martín Jiménez, V., \& Gil-Torres, A. (2017). Líderes políticos en YouTube: información y politainment en las elecciones generales de 2016 (26J) en España (Political leaders on YouTube: Information and politainment in the general elections 2016 in Spain). Profesional de la Información, 26(5), 937-946. https://doi.org/10.3145/epi.2017.sep.15

Bimber, B. (2003). Information and American Democracy: Technology in the Evolution of Political Power. Cambridge University Press. https://doi.org/10.1017/CBO9780511615573

Bimber, B. (2003). Information and American Democracy: Technology in the Evolution of Political Power. Cambridge University Press. https://doi.org/10.1017/CBO9780511615573

Blumenthal, S. (1980). The Permanent Campaign: Inside the World of Elite Political Operations. Beacon Press. 
Canel, M. J. (1999). Comunicación política (Political communication). Tecnos.

Capdevila, A. (1997). El diseño del spot de propaganda política: convergencia de estrategias comunicativas. Temes de disseny, (14), 193-199. Retrieved from http://www.raco.cat/index.php/Temes/article/view/29520/66434

Capdevila, A. (2002). El análisis del nuevo discurso político. Acercamiento metodológico al estudio del discurso persuasivo audiovisual (Doctoral dissertation, Universitat Pompeu Fabra). Retrieved from https://repositori.upf.edu/handle/10230/11781

Capdevila, A. Moragas-Fernández, C., \& Montagut, M- (2019). Viajes, juegos y guerras. Cómo los partidos políticos conceptualizan el proceso de independencia de Catalunya (Travel, games and wars. How political parties conceptualize the process of independence in Catalonia). In N. Pellisser \& J. M. Oleaque (Coords.), Mutaciones discursivas en el siglo XXI: la politica en los medios $y$ las redes (Discursive mutations in the 21st century: politics in the media and networks) (pp.113-140). Tirant lo Blanch.

Carceller Cobos, C. J. (2013). La videopolítica en campaña: evolución del spot electoral en España entre 2004-2011 (Videopolitics in campaign: evolution of the electoral spot in Spain between 2004-2011). Revista de Comunicación Vivat Academia, (124), 1-20. https://doi.org/10.15178/va.2013.124.1-20

Castells, M. (2008). Communication, Power and Counterpower in the Network Society (I). Telos, 74. Retrieved from https://telos.fundaciontelefonica.com/archivo/numero074/ communication-power-and-counterpower-in-the-network-society-i/

Congosto, M. L, Moro, E., \& Fernández, M. (2011). Twitter y política: información, opinión y ¿predicción? Cuadernos Evoca, (4), 11-15. Retrieved from http://evocaimagen.com/cuadernos/cuadernos4.pdf

Connolly-Ahern, C. \& Herrero, J. C. (2006). Political Advertising in Spain and Portugal. In L. L. Kaid \& C. Holtz-Bacha (Eds.), The SAGE Handbook of Political Advertising. SAGE. https:// doi.org/10.4135/9781412973403

Costa, P.-O. (2009). La utilització d'internet per part de Barack Obama transforma la comunicació política (Barack Obama's internet utilization transforms political communication). Quaderns del CAC, (33), 35-41. Retrieved from https://ddd.uab.cat/pub/artpub/2009/107699/quacac_ a2009m12n33p35.pdf

Devlin, L. P. (1989). Contrasts in presidential campaign commercials of 1984. American Behavioral Scientist, 32(4), 389-414. https://doi.org/10.1177/0002764289032004006

Diamond, E. \& Bates, S. (1984). The Spot. The Rise of Political Advertising on Television. The MIT Press.

Enguix Oliver, S. (2017). Impacto político e informativo de las redes sociales: esferas de actuación y comparación con los medios (Political and informative impact of social media: fields of action and comparison with the media). Anàlisi. Quaderns de Comunicació i Cultura, (56), 71-85. https://doi.org/10.5565/rev/analisi.3090

Gallardo-Paúls, B. (2014). Usos políticos del lenguaje. Un discurso paradójico (Political uses of language. A paradoxical speech). Anthropos.

Gallardo-Paúls, B. \& Enguix Oliver, S. (2016). Pseudopolítica: el discurso político en las redes sociales (Pseudopolitics: political discourse on social media). Dept Teoria dels Llenguatges i Ciències de la Comunicació-UV. 
García Beaudoux, V., D'Adamo, O., \& Slavinsky, G. (2007). Comunicación política y campañas electorales (Political communication and electoral campaigns). Gedisa.

García-Beaudoux, V. \& D'Adamo, O. (2007). El anuncio político televisivo como herramienta de comunicación electoral. Análisis de caso: los anuncios de la campaña para las elecciones legislativas de marzo de 2004 en España (Televised political spots as electoral communication tools. Case analysis: The spots used in the legislative election campaign in March, 2004, in Spain). Revista de Psicología Social, 22(1), 45-61. https://doi.org/10.1174/021347407779697502

Gavaldà, J. (1998). El ritme del relat audiovisual (The audiovisual tale ryhtm). In V. Alonso, A. Bernal, \& C. Gregori (Eds.), Actes del I Simposi Internacional de Narrativa Breu (Proceedings of the I Breu International Narrative Symposium) (pp. 177-196). Publicacions de l'Abadia de Montserrat.

Gil-Ramírez, M., Castillero Ostio, E., \& Gómez de Travesedo, R. (2020). Imagen y título de portada como elementos estratégicos de comunicación política en YouTube: uso por parte de la esfera política española (Image and cover title as strategic elements of political communication on YouTube: use by the Spanish political sphere). adComunica. Revista Científica del Estrategias, Tendencias e Innovación en Comunicación, 20, 255-282. https://doi.org/10.6035/2174-0992.2020.20.11

Gil-Ramírez, M. \& Gómez-de-Travesedo, R. (2020). Gestión de la política española en YouTube. Una asignatura pendiente (Management of Spanish politics on YouTube. A pending issue). Observatorio (OBS*), 14(1), 22-44. https://doi.org/10.15847/ obsOBS14120201491

Gil-Ramírez, M., Gómez-de-Travesedo, R., \& Almansa-Martínez, A. (2020). Debate político en YouTube: ¿revitalización o degradación de la deliberación democrática? (Political debate on YouTube: revitalization or degradation of democratic deliberation?) Profesional de la Información, 29(6). https://doi.org/10.3145/epi.2020.nov.38

Gómez, L. \& Capdevila, A. (2011). Ciutadans, polítics i democràcia: anàlisi retoricopersuasiva dels espots emesos a Catalunya (2008-2010) (Citizens, politics, and democracy. Persuasive rhetorical analysis of the electoral spots sent to Catalonia (2008-2010)). Comunicación: revista de recerca i anàlisi, 28(2), 27-46. https://doi.org/ 10.2436/20.3008.01.83

Gómez, L. \& Capdevila, A. (2012). Variaciones estratégicas en los spots electorales de televisión y de Internet en campaña electoral (Strategic variations in the electoral spots of Television and Internet in electoral campaign). ZER- Revista de Estudios de Comunicación, 17(33), 67-86. Retrieved from http://www.ehu.eus/ojs/index.php/Zer/article/view/10615/9855

Gueorguieva, V. (2007). Voters, MySpace, and YouTube. Social Science Computer Review, 26(3), 288-300. https://doi.org/10.1177/0894439307305636

Herrero, J. C. \& Connolly-Ahern, C. (2004). Origen y evolución de la propaganda política en la España democrática (1975-2000): Análisis de las técnicas y de los mensajes en las elecciones generales del año 2000 (Origin and evolution of political propaganda in democratic Spain (1975-2000): Analysis of the techniques and messages in the general elections of the year 2000). Doxa Comunicación. Revista interdisciplinar de estudios de Comunicación y Ciencias Sociales, (2), 151-172. Retrieved from http://hdl.handle.net/10637/6008

Holtz-Bacha, C. \& Kaid, L. L. (1995). A Comparative Perspective on Political Advertising: Media and Political System Characteristics. In Kaid, L. L. \& Holtz-Bacha, C. (Eds.), Political Advertising in Western Democracies. Parties y Candidates on Television (pp. 8-18). SAGE. 
Igartúa Perosanz, J. J. (2006). Métodos cuantitativos de investigación en comunicación (Quantitative research methods in communication). Bosch.

Innerarity, D. (2016). La política en la era de las redes (Politics in the networks era). Beerderberg. Retrieved from https://beersandpolitics.com/la-politica-en-la-era-de-las-redes

Jerit, J. (2004). Survival of the Fittest: Rhetoric During the Course of an Election Campaign. Political psychology, 25(4), 563-575. https://doi.org/10.1111/j.1467-9221.2004.00387.x

Kaid, L. L. \& Sanders, K. R. (1978). Political Television Commercials: An Experimental Study of Type and Length.Communication Research, 5(1), 57-70. https://doi.org/10.1177/009365027800500103

Kaid, L. L. (1999). Political advertising: A summary of research findings. In B. I. Newman (Ed.), Handbook of political marketing (pp. 423-438). SAGE.

Kaid, L. L. (2004). Handbook of political communication research. Lawrence Erlbaum Associates.

Kaid, L. L. \& Holtz-Bacha, C. (1995). Political advertising in Western democracies : parties and candidates on television. SAGE.

Kaid, L. L. \& Holtz-Bacha, C. (2006). Television advertising and democratic systems around the world: a comparison of videostyle content and effects. In L. L. Kaid \& C. Holtz-Bacha (Eds.), The SAGE Handbook of Political Advertising (pp. 445-458). SAGE.

Lipovetsky, G. (1990). El imperio de lo efimero. La moda y su destino en las sociedades modernas (The empire of the ephemeral. the fashion and its destiny in the modern societies). Anagrama.

Ley Orgánica 5/1985, de 19 de junio, del Régimen Electoral General 147 (Organic Law 5/1985, of June 19, on the General Electoral Regime 147) (Madrid) Spain). Retrieved from https://www.boe.es/boe/dias/1985/06/20/pdfs/A19110-19134.pdf

Ley Orgánica 2/1988, de 3 de mayo, reguladora de la publicidad electoral en emisoras de televisión privada (Organic Law 2/1988, of May 3, regulating electoral advertising on private television stations) (Madrid) (Spain). Retrieved from https://www.boe.es/boe/dias/1988/05/05/ pdfs/A13666-13666.pdf

Ley Orgánica 13/1994, de 30 de marzo, de modificación de la Ley Orgánica 5/1985, de 19 de junio, del Régimen Electoral General (Organic Law 13/1994, of March 30, modifying Organic Law 5/1985, of June 19, of the General Electoral Regime). Retrieved from https://www.boe.es/boe/dias/1994/03/31/pdfs/A10253-10256.pdf

Ley Orgánica 8/1999, de 21 de abril, de modificación de la Ley Orgánica 5/1985, de 19 de junio, del Régimen Electoral General (Organic Law 8/1999, of April 21, modifying Organic Law 5/1985, of June 19, of the General Electoral Regime). Retrieved from https://www.boe.es/boe/dias/1999/04/22/pdfs/A14924-14926.pdf

Ley Orgánica 6/2002, de 27 de junio, de Partidos Políticos (Organic Law 6/2002, of June 27, on Political Parties). Retrieved from https://www.boe.es/buscar/pdf/2002/BOE-A-200212756-consolidado.pdf

Ley Orgánica 2/2011, de 28 de enero, por la que se modifica la Ley Orgánica 5/1985, de 19 de junio, del Régimen Electoral General (Organic Law 2/2011, of January 28, which modifies Organic Law 5/1985, of June 19, of the General Electoral Regime). Retrieved from https://www.boe.es/boe/dias/2011/01/29/pdfs/BOE-A-2011-1639.pdf 
Lupia, A. \& Philpot, T. S. (2005). Views from Inside the Net: How Websites Affect Young Adults' Political Interest. Journal of Politics, 67(4), 1122-1142. https://doi.org/10.1111/j.14682508.2005.00353.x

Mattelart, A. (2000). La publicidad (Advertising). Paidós.

Negredo, S., Amoedo, A., Vara-Miguel, A., Moreno, E., \& Kaufmann, J. (2020). Resumen ejecutivo / DigitalNewsReport.es 2020: Decrece la confianza en la información, sube el pago por noticias digitales y aumenta el dominio del móvil (Executive summary / DigitalNewsReport.es 2020: Confidence in information decreases, payment for digital news rises and mobile dominance increases). Digital News Reports.es Retrieved from https://www.digitalnewsreport.es/resumen-ejecutivo-digitalnewsreport-es-2020decrece-la-confianza-en-la-informacion-sube-el-pago-por-noticias-digitales-yaumenta-el-dominio-del-movil/

Pellisser, N., Villar-Hernández, P., \& Enguix Oliver, S. (2017, April 27-28). El vídeo electoral: nuevas estrategias y nuevos mensajes en Twitter (The electoral video: new strategies and new messages on Twitter). Communication presented at the VI International Congress of Audio-visual Researchers Technologies and Digital Contents. Madrid, Spain.

Pericot, J. \& Capdevila, A. (2001). La evolución del discurso persuasivo electoral en televisión (The evolution of persuasive electoral discourse on television). Formats: revista de comunicació audiovisual, 3. Retrieved from http://www.raco.cat/index.php/Formats/ article/view/57029/343096

Peytibi, F. X., Rodríguez, J. A., \& Gutiérrez-Rubí, A. (2008). L'experiencia de les eleccions generals 2008 (The experience of the 2008 General Elections). Revista d'Internet, Dret $i$ Política, (7), 1-12. Retrieved from https://raco.cat/index.php/IDP/article/view/129985

Peña Jiménez, P. (2010). El spot político en España análisis pragmático comunicativo (The political spot in Spain communicative pragmatic analysis). Communication presented at the Communication and development in the digital age Congress. II AE-IC Congress 3, 4 and 5 February 2010, Malaga.

Peña Jiménez, P. (2011). El spot electoral negativo (The negative election advertising). Revista Latina de Comunicación Social, 66, 399-425. https://doi.org/10.4185/RLCS-66-2011-939-399-425

Peña Jiménez, P. (2013). El vídeo electoral como estrategia persuasiva en la campaña de 2011 (The electoral video as a persuasive strategy in the 2011 campaign). In I. Crespo Martínez (Ed.), Partidos, medios y electores en procesos de cambio. Las elecciones generales españolas de 2011 (Parties, media, and voters in processes of change. The 2011 Spanish general elections). Tirant Humanidades.

Peña Jiménez, P. \& García Jiménez, A. (2010). Tipología del spot electoral: una aproximación a partir de la campaña de 2008 (Typology of the election campaign spot: an approach from the 2008 campaign). Pensar la Publicidad, 4(2), 51-70. Retrieved from hhhttps://revistas.ucm.es/index.php/PEPU/article/view/PEPU1010220051A

Pineda, A., Garrido, M., \& Ramos, M. (2013). Análisis comparativo de la publicidad política en las elecciones de 2008 de Estados Unidos y España (A comparative Analysis of American and Spanish Political Advertising in 2008 Presidential Elections). Zer: Revista de estudios de comunicación= Komunikazioikasketenaldizkaria, 18(34),73-91. https://doi.org/10.1387/zer.10645 
Propaganda electoral de los líderes en las "elecciones de 1977. (n.d.). rtve.es. Retrieved from https://www.rtve.es/play/videos/fue-noticia-en-el-archivo-de-rtve/propagandaelectoral-lideres-elecciones-1977/2344067/

Rodríguez-Breijo, V., Gallardo-Camacho, J., \& Sierra-Sánchez, J. (2018). Información política en los vídeos que son tendencia en YouTube España (Political information in trending topic videos on YouTube Spain). El Profesional de la Información, 27(5), 1041-1049. https://doi.org/10.3145/epi.2018.sep.08

Sádaba, T. (2003). Los anuncios de los partidos en televisión. El caso de España (1993-2000) (Political parties' advertisements on TV. The case of Spain (1993-2000)). In S. Berrocal (Ed.), Comunicación política en televisión y nuevos medios (Political communication on TV and new media) (pp. 163-205). Ariel.

Sádaba, T. \& Jové, M. (2017). Political Advertising in Spain (1977-2015): From Education to Indirect Effects. In C. Holtz-Bacha \& M. R. Just (Eds.), Routledge Handbook of Political Advertising. Taylor and Francis.

San Martí, J. M. (2003). Los nuevos agentes políticos en la comunicación televisiva (The new political agents in television communication). In S. Berrocal Gonzalo (Ed.), Comunicación política en televisión y nuevos medios (Political communication on TV and new media) (pp. 81-109). Ariel.

Serazio, M. (2014). The New Media Designs of Political Consultants: Campaign Production in a Fragmented Era. Journal of Communication, 64(4), 743-763. https://doi.org/10.1111/jcom.12078

Stanyer, J. (2005). Political Parties, the Internet and the 2005 General Election: From Web Presence to E-Campaigning? Journal of Marketing Management, 21(9-10), 1049-1065. https://doi.org/10.1362/026725705775194094

Toffler, A. (1980). La tercera ola (The Third Wave). Plaza y Janés.

Vesnic-Alujevic, L. \& Van Bauwel, S. (2014). YouTube: A Political Advertising Tool? A Case Study of the Use of YouTube in the Campaign for the European Parliament Elections. Journal of Political Marketing, 13(3), 195-212. https://doi.org/10.1080/15377857.2014.929886

Weber, L. M., Loumakis, A., \& Bergman, J. (2003). Who Participates and Why? Social Science Computer Review, 21(1), 26-42. https://doi.org/10.1177/0894439302238969

Wood, S. C. (1990). Television's First Political Spot Ad Campaign: Eisenhower Answers America. Presidential Studies Quarterly, 20(2), 265-283. Retrieved from https://www.jstor. org/stable/27550614 


\section{SOBRE LOS AUTORES}

PAZ VILLAR-HERNÁNDEZ, doctora por la Universitat de València. Licenciada en Periodismo (Ciencias de la Información) por la Universitat Politècnica de València y Máster en Educación y Tecnologías de la Información y la Comunicación por la Universitat Oberta de Catalunya. En la actualidad trabaja como técnico de formación permanente en el Servei de Formació Permanent i Innovació Educativa de la Universitat de València. Es co-editora de la revista científica, Research in Education and Learning Innovation Archives. Trabajó como docente en Duke University (Estados Unidos).

NEL-LO PELLISSER ROSSELL, profesor titular de Comunicación Audiovisual de la Universidad de València. Docente en el máster de Producción de Contenidos y formatos audiovisuales de la UV, en el máster de Comunicación Corporativa Integral de la Universitat Ramon Llull y en el Postgrado de Periodismo científico de la UNED. Las principales líneas de investigación se centran en el análisis de los discursos mediáticos. Los últimos trabajos publicados tienen como objeto el estudio del discurso político en las redes. 